\title{
Intelligent system for lighting control in smart cities
}

\author{
Juan F. De Paz ${ }^{a}$, Javier Bajo ${ }^{\text {b,*, Sara Rodríguez }}{ }^{a}$, Gabriel Villarrubia ${ }^{a}$, \\ Juan M. Corchado ${ }^{\mathrm{a}}$ \\ ${ }^{a}$ Department of Computer Science and Automation, School of Science University of Salamanca. Plaza de la Merced s/n, 37008, Salamanca, \\ Spain \\ ${ }^{\mathrm{b}}$ Department Artificial Intelligence, School of Computer Science, Polytechnic University of Madrid. Campus Montegancedo, Boadilla del \\ Monte, Madrid, 28660, Spain
}

\section{A R T I C L E I N F O}

\section{Article history:}

Received 18 October 2015

Revised 31 July 2016

Accepted 14 August 2016

Available online 17 August 2016

\section{Keywords:}

Light sensors

Intelligent systems

Distributed systems

Autonomous control

Street lighting

\begin{abstract}
A B S T R A C T
This paper presents an adaptive architecture that centralizes the control of public lighting and intelligent management to economize lighting and maintain maximum visual comfort in illuminated areas. To carry out this management, the architecture merges various techniques of artificial intelligence (AI) and statistics such as artificial neural networks (ANN), multi-agent systems (MAS), EM algorithm, methods based on ANOVA, and a Service Oriented Approach (SOA). It achieves optimization in terms of both energy consumption and cost by using a modular architecture, and is fully adaptable to current lighting systems. The architecture was successfully tested and validated and continues to be in development.
\end{abstract}

(c) 2016 Elsevier Inc. All rights reserved.

\section{Introduction}

The concept of Smart Cities is an increasingly common trend in technology-based projects. Balancing the environment and natural resources is a practical and responsible key for these paradigms, which aim to increase the comfort in the daily lives of citizens and institutions by applying sustainable development practices. One of the goals of the systems developed under the concept of Smart Cities is to apply new technologies to obtain sustainable economic development and provide better quality of life. We have used the system presented in this study to produce a distributed lighting system to facilitate the implementation of a new infrastructure in a city; in other words, we have developed a modular architecture that is fully adaptable to a city's existing lighting systems and, consequently, optimizes energy consumption and costs. The subject of energy efficiency is paramount not only to reduce energy costs, but also to promote environmental and economic sustainability.

One of the greatest expenses that town and city councilmembers must contemplate is the lighting bill. According to IDAE [16], there were about 4,800,000 points of light in Spain during 2010, with an average power of $180 \mathrm{~W}$ and $4200 \mathrm{~h}$ of annual use. Representing an electricity consumption of $3630 \mathrm{GWh} /$ year, this usage is considered excessive. Technological advances in external lighting installations, along with their intelligent use, will make it possible to reduce our overall consumption.

This research appears as part of a greater project by the BISITE (Bioinformatics, Intelligent Systems and Technology Education) research group of the University of Salamanca, which is to build a system that can centralize the control and

\footnotetext{
* Corresponding author.

E-mail addresses: fcofds@usal.es (J.F. De Paz), jbajo@fi.upm.es (J. Bajo), srg@usal.es (S. Rodríguez), gvg@usal.es (G. Villarrubia), corchado@usal.es (J.M. Corchado).
} 
intelligent management of street lighting in order to reducing lighting costs and maintain maximum visual comfort in illuminated areas. This will serve to avoid excessive illumination of areas, as it is often not necessary to maintain maximum light intensity to provide optimal service.

To validate the system in an experimental research level, a small test case is available, consisting of a portable installation of five luminaires with specific hardware. The target functionality is divided into two modules. One module will be responsible for the direct management of the various installations and control panels, and will serve as a communication layer with each site, optimizing the ability to control and monitor each facility and luminaire in almost real-time. To this end, the system must provide a service interface that can be accessed for each installation using a standardized interface, independent of the underlying technology and hardware of each installation.

The main objective of the second module will be to manage the lighting schedule, consumption, and prediction for each installation. For the purposes of our study, a light plan is defined as the light output level for each installation offered hourly. It must be possible to establish this light plan by programming user preferences, or by observing different decisive environment factors in determining the appropriate level of brightness for each site at each time. Thus, different factors must come into play: an astronomical clock, weather and traffic, and pedestrian flow. Moreover, it should be possible to predict consumption by the light patterns assigned to each zone, depending on the economic rate.

In order to manage these various elements, the system built for this study combines different statistics and artificial intelligence (AI) techniques such as artificial neural networks (ANN), EM algorithm, methods based on ANOVA and a Service Oriented Approach (SOA) [8]. The combination of these techniques makes the system innovative in terms of obtaining an intelligent prediction of consumption and cost. The innovative part of the system presented is the combination of these methods in a workflow that can automatically obtain smart lighting schedules based on input data such as flow of pedestrians or traffic, weather, or the cost of consumption associated with luminaires.

The article is structured as follows: Section 2 presents the state of the art for projects and research conducted in the field of Smart cities and light control, showing the most commonly used techniques in this field, and comparing these techniques with the system presented. Section 3 introduces the presented system, its operation and details of the techniques used. Section 4 describes the case study developed for system validation, and finally, Section 5 provides some results and conclusions of this work.

\section{Background}

The concept of smart cities, smart environments, or smart homes [14] is still emerging in our society. One of the many definitions of Smart Cities is: "The use of smart computing technologies to make city services more intelligent, interconnected and efficient - which includes administration, education, health care, public safety, real estate, transportation and utilities." [23]. It seems clear that the purpose of these cities is sustainable economic development, based on new technologies (ICT) to provide better quality of life and prudent management of natural resources through the engagement of all citizens. To make a "smart" city is one of the objectives most often identified in current research as a strategy to mitigate the problems caused by the rapid growth of urban populations. Problems such as lack of resources, pollution, traffic congestion and deteriorating infrastructure are some of the many problems that large urban populations increasingly face [6].

Within fields related to energy efficiency, more and more cities around the world are now committed to the development of pilot projects $[3,17]$, including Spain, with its Málaga Smart City, a project that aims to save energy by micro power management. This includes energy storage in batteries for use in buildings, street lighting and electrical transport, promoting the use of electric cars, etc. Another example is Smart City Valladolid-Palencia, a joint venture between two cities whose objective is to manage a Smart city while overcoming the obstacle of distance that separates them. The program aims to implement a smart meter network, integrate electric cars, increase energy efficiency in buildings, organize traffic, etc.

In an effort to optimize energy efficiency through lighting management, this research includes the installation and control of distributed lighting systems to facilitate the implementation of a new infrastructure in a city or the optimization of existing infrastructure; further integration with other control systems, and optimization of heating, cooling or controlling air quality $[9,12,21,22]$. Other studies have addressed the issue of energy efficiency, offering specific applications. For instance, the authors in [1] present a systematic approach to the modeling, optimization, control, and adaptation of a color-tunable LED lighting control system. Through light sensor feedback, the control system is able to achieve significant energy savings without substantially sacrificing lighting quality. The key techniques used here are an appropriate choice of cost function based on color metrics and the trade-off between quality of light and energy consumption for LED lighting systems.

The authors in [24] employ formal methods to design a graph model, accompanied by various means of control, including AI methods (rule-based systems, pattern matching) to design and control an outdoor lighting system. In this case, the work is focused only on the design phase and the control phase designing features such as dynamic, sensor-based control, multiple luminaire states and complex geometries. Other research on lighting control systems base their operation in image processing [5], fuzzy systems [25], cooperative methods and wireless sensor network (WSN) [26] or simulation algorithms [4] and predictive control [2] for energy optimization.

There are also some tools already developed, such as Lites, which has temperature sensors, ambient light, power, motion detection; CityLight, which allows the remote management of lighting, fault detection and planning lighting patterns manually, or Tvilight, which regulates the lighting based on presence sensors and maintains minimum brightness in inactive hours. 


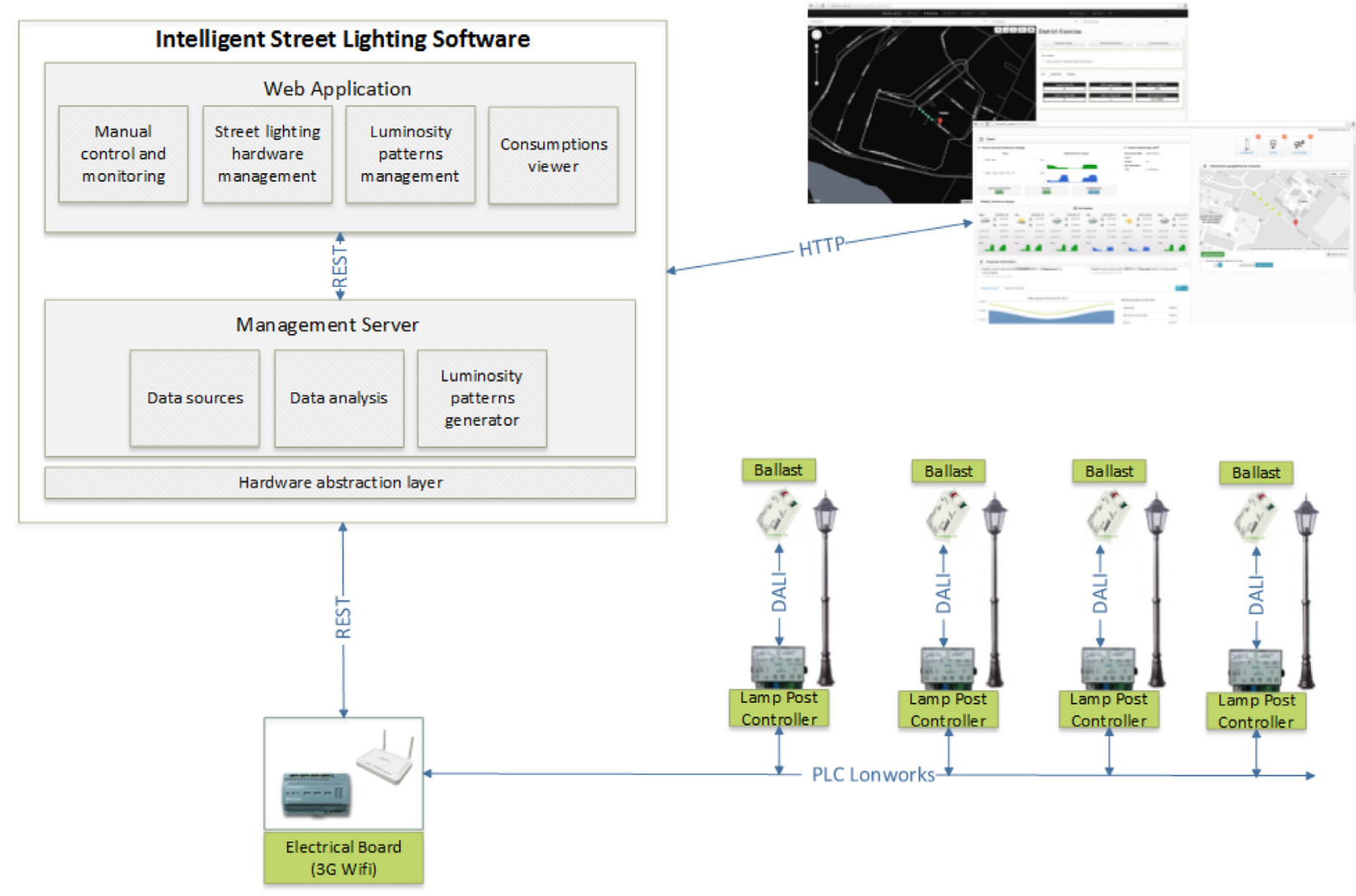

Fig. 1. Main system components.

Given the current research presented, it is possible to observe that, at present, there is no system or architecture that remotely manages a lighting system, regardless of the particular system used, and allows for intelligent management, optimizing both consumption and its associated costs. This paper presents an adaptive architecture that allows the centralized control of numerous public lighting installations [13]. Specifically, it allows distributed and real-time intelligent control based on prediction and analysis techniques of lighting, one of the main shortcomings of the systems listed above. From a modular architecture fully adaptable to the current lighting systems, energy and economic optimization is possible. The architecture has been successfully tested and validated and continues to be in development today. The following sections describe the operation and technologies used in the architecture and the results currently obtained.

\section{Proposed architecture}

The system presented in this paper aims to frame the intelligent management of all public lighting, including monitoring and real-time control of the lights, and to establish lighting patterns that fit with the use of the public highway installations.

Fig. 1 shows the context of the system, mainly composed of the control software (Intelligent Street Lighting Software) and the set of public-lighting installations, accessible via the internet. Facilities include special hardware for global and individual control of each luminaire, while communication between devices is done by PLC. The control software is composed of three modules:

- The hardware abstraction layer allows communication with facilities regardless of the underlying hardware.

- The management server contains both device management and intelligent algorithms for efficient energy management. The "Data sources" module captures information related to pedestrian and traffic flow, weather data, and data about the monitoring of the facility. The "Data analysis" module deals with the study of information collected for the detection of foot traffic patterns, management of neural networks to predict consumption from light intensities, and estimates of consumption online. Finally, the "Luminosity patterns generator" module allows the creation of adequate light planning suitable for the specific facility lighting depending on the standards of pedestrian flow and weather conditions of each case.

- The web application provides access to all functionalities for configuring lighting schedules, monitoring and controlling facilities. 


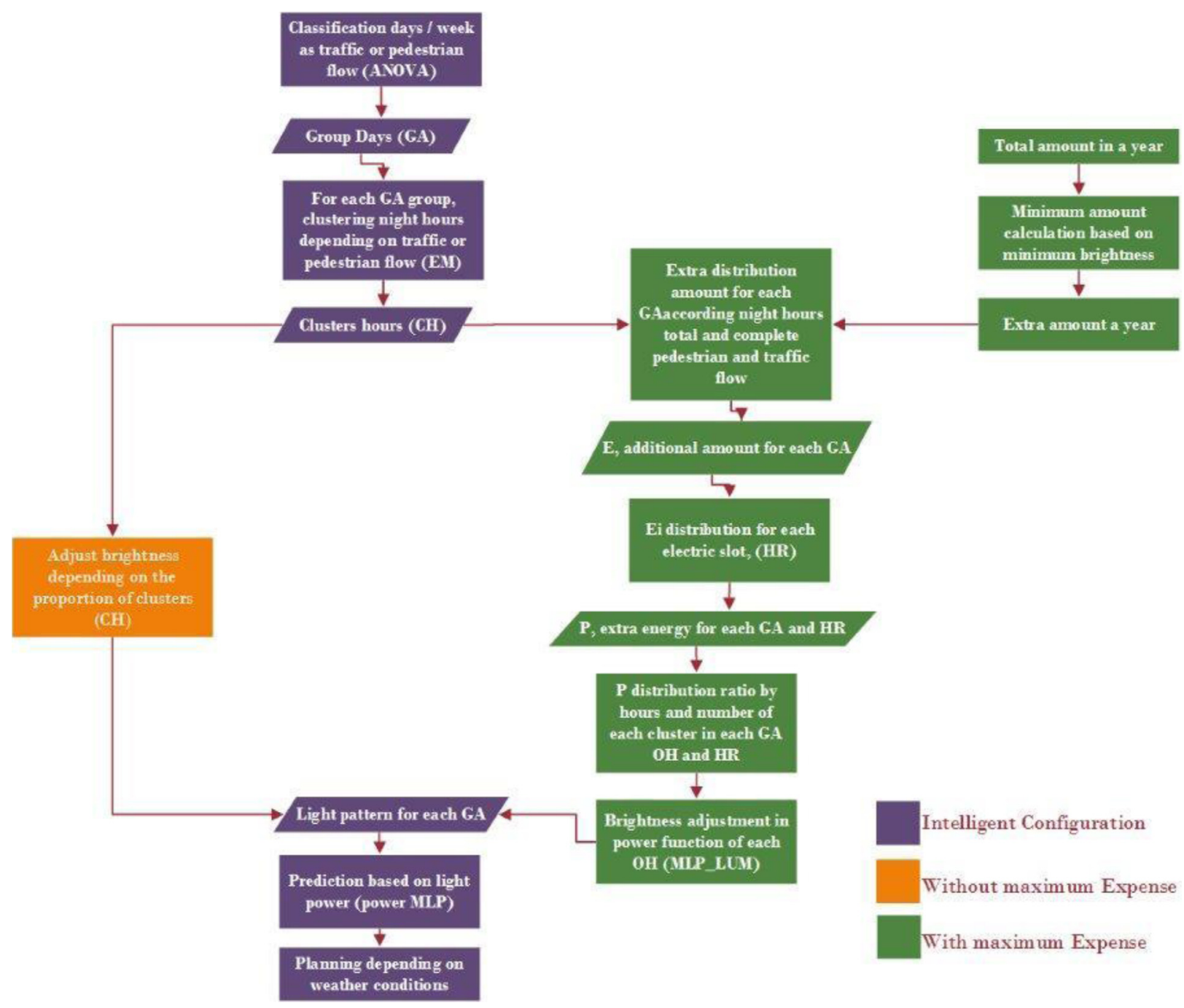

Fig. 2. Workflow procedures followed for lighting.

The ideal goal of the street lighting architecture design is to work well and provide safe and stable street lighting control for our daily life without human intervention. However, as human users should know whether the system is working normally, the interaction between the system and human users is necessary. There are also specific situations in which the system should be manually controlled by human users. The system includes the ability to automatically ("smartly") or manually interact according to the lighting used and the needs of the specific case study.

\subsection{Workflow}

The establishment of adequate lighting configuration for each environment is one of the objectives of this project. Light configuration refers to a set of times at which the area is illuminated according to the brightness levels associated with each time of day. This will save on lighting consumption, maintaining a state of maximum visual comfort in the lighted areas, as there are many occasions where it is not necessary to maintain a maximum level of light intensity to provide optimal service to the area, which generally causes excessive consumption.

In the presented system, the light designs can be set either manually or through a smart process. In the former case, the user is encouraged to plan the time slots (in hours) and the luminous flux of each time slot. In the latter case, we proceed to the observation of different environmental factors that may be influential in determining adequate lighting for the particular area, such as flow or pedestrian traffic, or weather conditions, which influence the level of ambient light, especially near the hours of sunrise and sunset.

The flow diagram of Fig. 2 shows the procedures to complete the light patterns according to ambient factors and different user preferences. It is possible to observe two different workflows, which correspond to the process of generating light 


\begin{tabular}{|c|c|c|c|c|}
\hline \multicolumn{1}{|c|}{$\beta$} & Monday & Thuesday & $\ldots$ & Sunday \\
\hline Interval 1 & $\mathrm{y}_{111}, \mathrm{y}_{112}, \mathrm{y}_{113}$ & $\mathrm{y}_{121}, \mathrm{y}_{122}, \mathrm{y}_{123}$ & $\ldots$ & $\mathrm{y}_{1 J 1}, \mathrm{y}_{172}, \mathrm{y}_{1 J 3}$ \\
\hline Interval 2 & $\mathrm{y}_{211}, \mathrm{y}_{212}, \mathrm{y}_{213}$ & $\mathrm{y}_{221}, \mathrm{y}_{222}, \mathrm{y}_{223}$ & $\ldots$ & $\mathrm{y}_{2 J 1}, \mathrm{y}_{2 J 2}, \mathrm{y}_{2 J 3}$ \\
\hline$\ldots$ & $\ldots$ & $\ldots$ & $\ldots$ \\
\hline Interval I & $\mathrm{y}_{111}, \mathrm{y}_{112}, \mathrm{y}_{113}$ & $\mathrm{y}_{121}, \mathrm{y}_{122}, \mathrm{y}_{123}$ & $\ldots$ & $\mathrm{y}_{I J 1}, \mathrm{y}_{I J 2}, \mathrm{y}_{\mathrm{IJ3}}$ \\
\hline
\end{tabular}

Fig. 3. Table with the data from ANOVA.

patterns for a given period of time. One of the flows can generate patterns without establishing a maximum estimated expenditure, while the other can set it. Maximum expenditure means the maximum amount to spend on the lighting bill for the period over which the light patterns are concluded.

Both processes share the initial logic. The first step gathers historical data of pedestrian and traffic flow over several weeks. Subsequently, a classification is performed by analysis of variance (ANOVA) [20] to determine on what day of the week the patterns share pedestrian and traffic flow according to the different hours of the day.

After obtaining the groups of days, a clustering algorithm (Expectation-maximization EM) [11,19] is applied to each group in order to determine the time of night when there tends to be a similar number of pedestrians or traffic.

After these two steps, the flowchart shows a bifurcation. The left branch corresponds to the process to follow if a maximum expenditure has not been set, which mainly follows a simple process of adjusting lighting levels based on the proportion of generated clusters after EM technique for each group of days. The upper branch applies the logic to be followed when a maximum flow is established. The steps followed in this last branch focus primarily on the optimal distribution of illumination and the specific times when the system has to provide the same light levels in scenarios with similar environmental characteristics. The distribution amount is performed according to features such as hourly rate, with or without time restrictions, the evening hours that can affect lighting needs, and the proportion of clusters based on traffic and pedestrian flows.

The two branches obtain different light patterns depending on the groups of days generated with the ANOVA technique. The penultimate step in the flowchart consists of predicting the cost of the completed lighting design, which in the case of the branch with maximum expenditure, will coincide with a small margin of error depending on the expenditure estimation technique used. The estimate of expenditure is performed by a neural network MLP (Multi-Layer Perceptron) [15,7], which predicts power level as a function of lumens and is trained with historical data of the type of luminaire used in each installation.

\subsection{Days classification and clustering}

In order to carry out the light distribution during the different days in a week, it is necessary to apply a search pattern to differentiate the days. To determine the set of days with similar characteristics, several variables must be taken into account, such as day of the week, interval, and the volume of detected people. If the variables are not quantitative then it is necessary to apply clustering techniques such as ANOVA to extract similarities among groups. In this case, a two-way ANOVA with repetition is applied. The factors are the day of the week and the interval, which is considered a group factor. The information managed by ANOVA is shown in the Fig. 3. The values $y_{\mathrm{ijk}}$ are the volume of detected persons in the interval $\mathrm{i}$ and day $\mathrm{j}$ in the week $\mathrm{y}$ and repetition $\mathrm{k}$. The repetitions are measured in different weeks on the same day.

The regression model is defined by

$$
y_{i j k}=\mu+\alpha_{i}+\beta_{j}+(\alpha \beta)_{i j}+\mu_{i j k} \text { with } \mu_{i j k} \equiv N\left(0, \sigma^{2}\right) \text { independent }
$$

The F observed can be calculated according to (2) to determine the relevance of the relations in ANOVA.

$$
F_{\alpha \beta}=\frac{\sum_{i=1}^{I} \sum_{j=1}^{J} \sum_{k=1}^{K}\left(\bar{y}_{i j \bullet}-\bar{y}_{i \bullet \bullet}-\bar{y}_{\bullet j \bullet}+\bar{y}_{\bullet \bullet \bullet}\right)^{2} /(I-1)(J-1)}{\sum_{i=1}^{I} \sum_{j=1}^{J} \sum_{k=1}^{K} e_{i j k}^{2} / I J(K-1)}
$$




$$
F_{\beta}=\frac{K I \sum_{j=1}^{J}\left(\bar{y}_{\bullet j \bullet}-\bar{y}_{\bullet \bullet \bullet}\right)^{2} /(J-1)}{\sum_{i=1}^{I} \sum_{j=1}^{J} \sum_{k=1}^{K} e_{i j k}^{2} / I J(K-1)}
$$

Where

$$
\begin{aligned}
& \bar{y}_{\bullet \bullet \bullet}=\frac{1}{I J K} \sum_{i=1}^{I} \sum_{j=1}^{J} \sum_{k=1}^{K} y_{i j k} \\
& \bar{y}_{i \bullet \bullet}=\frac{1}{J K} \sum_{j=1}^{J} \sum_{k=1}^{K} y_{i j k} \\
& \bar{y}_{\bullet \bullet \bullet}=\frac{1}{I K} \sum_{i=1}^{I} \sum_{k=1}^{K} y_{i j k} \\
& \bar{y}_{i j \bullet}=\frac{1}{K} \sum_{k=1}^{K} y_{i j k} \\
& e_{i j k}=y_{i j k}-\bar{y}_{i j \bullet}
\end{aligned}
$$

Once $F_{\alpha \beta} F_{\beta}$ is calculated, it is possible to determine if a specific factor or the interaction between factors affects the model. If the interaction does not affect the model, then the volume of people/traffic does not affect the model. To determine the level of influence of the interaction, a contrast of hypothesis HO is performed: $(\alpha \beta)_{i j}=0 \forall i j$. The statistic of the contrast is defined when $\mathrm{HO}$ is rejected, which results in a difference between days and time slots.

$$
F_{\alpha \beta}>F_{(I-1)(J-1), I J(K-1), 1-\alpha}
$$

To determine if a specific day affects the model, it is necessary to carry out the contrast H0: $\beta_{j}=0 \forall j$. The statistic for the contrast is defined as reject $\mathrm{HO}$ and, thus (5) there is a difference between days.

$$
F_{\beta}>F_{(J-1), I J(K-1), 1-\alpha}
$$

The grouping process is based on finding those days of the week for which HO is not rejected in an iterative way. When it is rejected, however, a new group i is created, as shown in (7).

$$
\begin{aligned}
& g_{i}=\cup \beta_{j} / F_{\alpha \beta} \leq F_{(I-1)(J-1), I J(K-1), 1-\alpha} \cap F_{\beta} \leq F_{(J-1), I J(K-1), 1-\alpha} \\
& g_{i} \cap g_{j}=\varphi \\
& \cup g_{i}=\beta
\end{aligned}
$$

When the process finishes, we have $\mathrm{n}$ groups. The group $\mathrm{i}$ is defined as $\mathrm{g}_{\mathrm{i}}$ and every group contains the days of the week that are considered as equivalent for the planning process for distributing the luminosity.

For each of the identified groups $g_{i}$ a new grouping process is carried out based on the time slots taken into consideration and on the observed flow of people. To obtain the clusters, we apply the EM clustering technique [18]. The clustering process takes into account the number of people in each slot of time and the days that are assigned to group $g_{i}$.

\subsection{Approximation of power consumption}

To estimate the prediction of consumption and the corresponding cost for the lighting system, a neural network is used. The consumption level has a non-linear dependence with the luminosity level, making it necessary to create a mechanism to determine the level of consumption for the desired level of luminosity, and vice versa. In our proposal, we used a MLP neural network that incorporates bias for the training process. The number of neurons in the hidden layer is 7 and the activation function is sigmoidal. The weights of the neurons in the hidden and output layers correspond to those used in [18] or the previous work [10].

To carry out the training and the estimation of all the values, they are normalized in the [0.2- 0.8] interval, taking into account the activation function used in the network.

\subsection{Distribution of expenditure}

To calculate the distribution of the maximum $E_{T}$ in $Z$ time entered by the user, it is first necessary to calculate the minimum amount of expenditure $E_{\min }$ to a minimum brightness $L_{\min }$. This time period has $N h$ overnight hours. One MLP network is used to predict the spending power of the luminaires used depending on the required level of brightness. The additional expenditure $E$ will be distributed for generating light patterns.

$$
\begin{aligned}
& L_{\text {min }} \rightarrow R N A_{\text {pow }} \rightarrow \text { Pow }_{\text {min }} \\
& E_{\text {min }}=P o w_{\text {min }} N h \\
& E=E_{T}-E_{\text {min }}
\end{aligned}
$$




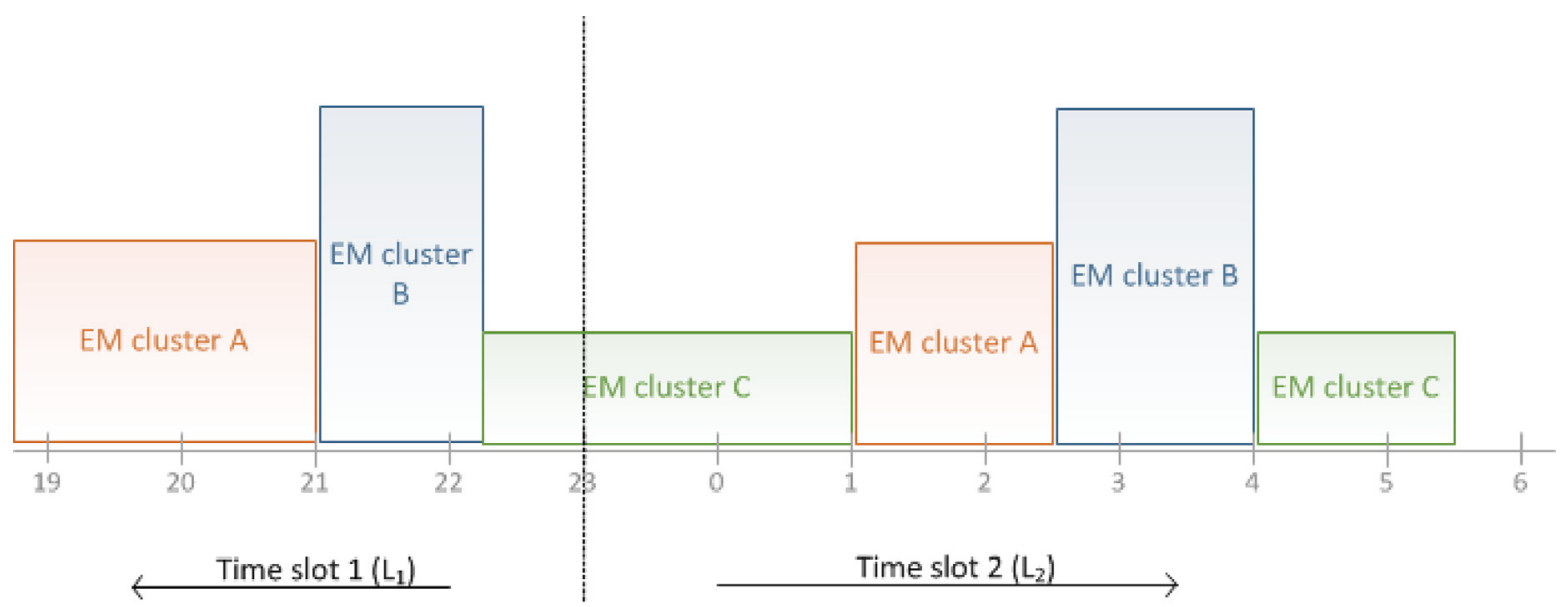

Fig. 4. Area of time slots.

The first step taken is to distribute the amount $E$ between groups of days $g_{i}$ generated in the ANOVA process. This distribution is based on the number of hours of night $N h_{i}$ of each group $i$ as well as the pedestrian and traffic flow $P_{i}$ that exists in that group.

The calculation of traffic and pedestrian flow $P_{i}$ of each $g_{i}$ is done by calculating the average number of people who pass through the area every night $\overline{P d_{l}}$ and the number of days $D_{i}$ that belong to $g_{i}$, taking the average of the historical data used before (Fig. 1). The number of people is limited and will be equal to the upper bound if the bound is exceeded.

$$
\begin{aligned}
P_{i} & =\overline{\operatorname{Pd}_{l}} D_{i} \\
\overline{P d_{l}} & =\frac{1}{J K} \sum_{i=1}^{I} \sum_{j=1}^{J} \sum_{k=1}^{K} y_{i j k} / j \in g_{i}
\end{aligned}
$$

Both $N h$ and $P$ variables can have different degrees of influence $\rho$ at the time of allocation of the amount $E$. The extra expense of each group $g_{i}$ is given by Eq. (10).

$$
E_{i}=\frac{N h_{i}}{N h} E \rho_{N h}+\frac{P_{i}}{P} E \rho_{p}
$$

Where:

$$
\begin{aligned}
& N h=\sum_{i=1}^{I} N h_{i} ; P=\sum_{i=1}^{I} P_{i} \\
& N h=\sum_{i=1}^{I} N h_{i} ; P=\sum_{i=1}^{I} P_{i} \\
& 0 \leq \rho_{N h} \leq 1 ; \quad 0 \leq \rho_{p} \leq 1 ; \quad \rho_{N h}+\rho_{p}=1
\end{aligned}
$$

Once the distribution of expenditure for each group is done, $E_{i}$, the distribution process is repeated, in this case between different times Fs of stating the electricity cost associated with the area to be illuminated. To do this, we take into account the proportion $\left(w_{i r}\right)$ and time of use in hours $\left(n_{i r}\right)$ for each group $m_{i r}$ (which represents the values that are classified in the $E M_{r}$ cluster of each group $g_{i}$ ANOVA), and the price of the energy in each time slot, $L_{s}$.

Fig. 4 shows a possible deployment scenario in which clusters $m_{i r}$ indicate the result of the EM algorithm, where each cluster is represented by a rectangle. The $\mathrm{x}$-axis represents time in hours, while the $\mathrm{y}$-axis represents the proportion of clusters $\left(w_{i r}\right)$, which is determined by the average pedestrian and traffic flow determined for each group $m_{i r}$. The distribution of expenditure $E_{i}$ is done by calculating the total area of each time slot $F_{S}$ indicating the weighted price of energy in these slots. Thus, we can ensure a fair distribution of the expenditure and can illuminate with the same schedules for light flow spaces as those used with similar environmental factors (10).

$$
\begin{aligned}
E_{i s} & =\left(\sum_{r=1}^{R_{i}} n_{i r} w_{i r}\right) L_{S} X \\
E_{i} & =\sum_{S=1}^{S} E_{i s}
\end{aligned}
$$


where

$$
w_{i r}=\frac{\sum_{i=1}^{I} \sum_{j=1}^{J} \sum_{k=1}^{K} y_{i j k}}{I J K} / j \in g_{i}, i \in m_{i r}
$$

Once the distribution of expenditure for each time slot $F_{S}$ of each group of days $g_{i}$ is made, the price of energy does not vary for each slot of the group. In this step, each expenditure $E_{i s}$ becomes equivalent to the energy consumed in the slot $s$ of the days that belong to group $i$ during the period of time $Z$, which the user will have chosen at the beginning of the process. The distribution of extra power (which will continue to be denoted as $E_{i s}$ ) for each hour $h \in H_{\text {irs }}$ (set of hours that belong to $F_{s}$ slot grouped in subgroup $m_{i r}$ hours, of the initial group $g_{i}$ ) was performed similarly to the previous step, to continue to ensure a fair distribution. It will consider both the size of cluster $w_{i r}$ as the number of hours used $n_{i r}$.

$$
\begin{aligned}
& p_{h}=w_{i r} x / h \in H_{i r s} \\
& E_{i s}=\left(\sum_{r=1}^{R_{i}} n_{i r} w_{i r}\right) \chi
\end{aligned}
$$

The expression (13) denotes $p_{h}$ as the extra power to supply each hour $h \in H_{\text {irs }}$. In this way, the power to supply in each hour to all luminaires $\left(p_{t h}\right)$ will be the minimum power (8) of each hour with more extra power calculated $p_{h}$.

$$
p_{t h}=\frac{P o w_{\min }}{N h}+p_{h}
$$

At this point, the power supply to each luminaire in every hour (simply dividing $p_{t h}$ by the number of luminaires) is known. For the resulting light output of the power supplied, an MLP network that predicts light output from input power is utilized. The equivalence between luminous flux and power tends to be linear, so this approximation is quite accurate: in most cases, doubling the power spent will result in twice the luminosity.

\section{Results}

To verify the validity of the system, a case study was conducted with an experimental prototype. To develop the first prototype a hardware solution installed in a briefcase was purchased. Fig. 5 shows a photo of the prototype environment that emulates an installation of five street lamps with a control node. Four interior and one exterior lamp were used to test the control system while maintaining low cost hardware. Each lamp is controlled by an adjustable ballast hidden in the top panel. A luminaire controller regulates and monitors each ballast and liaises with the control node.

Luminaire controllers (ISDE brand ASL-510-TCH) are placed inside each lamp and communicate via PLC with the control node, but can also be placed at the beginning of a line of street lamps. These controllers interpret commands received through the line to regulate the output of the ballasts of the lamps using the DALI protocol. Additionally, they monitor the status of the lamps, the instantaneous consumption, and the power supply of each lamppost.

The control node chosen for this study is an Echelon Smart Server, a general purpose controller that automates noncritical processes. In street lighting systems, it is possible to control and monitor up to 192 single or double lamps head through PLC. The system offers a SOAP interface for configuration and remote management that has been used for integration with the developed system. The PLC signal injected by the control node is replicated in three phases using a phase coupler [three-phase coupler PLC]. The network analyzer is the CVM-MINI brand model Circutor. It connects via a parallel port RS-485 to Echelon SmartServer, with which it communicates using the MODBUS protocol. For the prototype system that estimates pedestrian flow, we used an IP camera TP-LINK DSC-942L interior placed on a window.

Fig. 6 shows the basic schema of the deployment carried out in the street controlled by our system.

The content of the control panel is described in the following paragraph, and is similar to the development prototype. DALI ballasts are installed in each of the lamps, as well as a controller that liaises with the Smart Server Dashboard.

A light sensor was installed in the control panel to evaluate the ambient light conditions and light output provided by the lamps. An outdoor IP camera equipped with infrared vision (Foscam FI8905W) obtains the images that will be used to estimate the flow of pedestrians and traffic in the street.

Fig. 7 shows a diagram of the internal structure of the control panel. As can be seen in Fig. 7, a WiFi router/switch facilitates remote Access to the Smart Server and to the light sensor, making use of the communication infrastructure deployed in the village.

To construct the light sensor, we used an Arduino microcontroller with Ethernet connectivity and a TEMT6000 luminosity sensor. The sensor can be accessed through the communication network. Both the light sensor and the Smart Server are connected via Ethernet to the WiFi router.

All electricity installation is controlled through the control panel, where the control signal is injected via PLC using the phase coupler. The rest of the devices deployed for the case study are identical to those used in the development environment. The intelligent agents and the services were deployed in virtual machines in a private server installed in the University of Salamanca. 


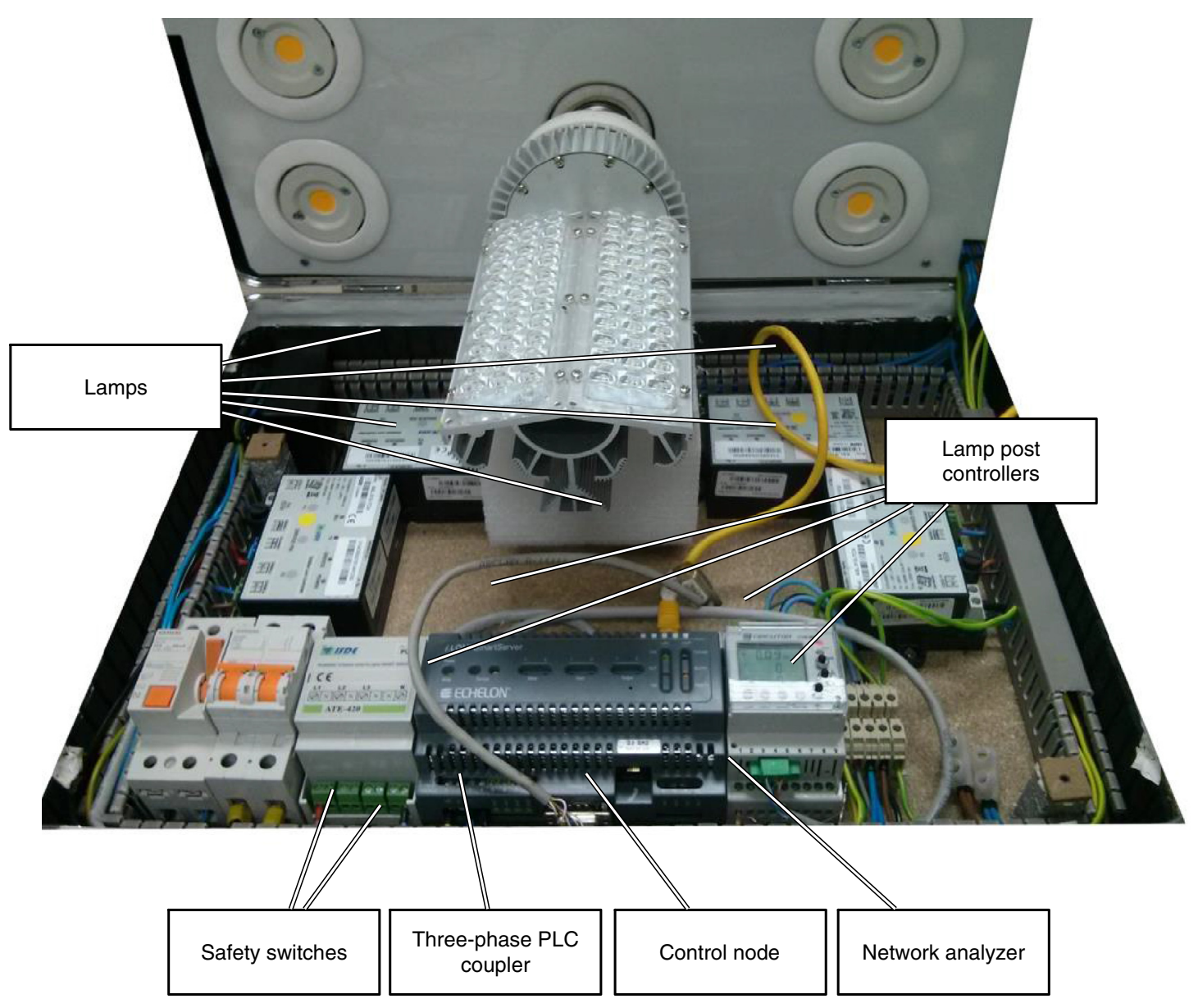

Fig. 5. Prototyping environment that simulates an installation of five streetlights.

To demonstrate the validity of the system, and consequently, the methods used in Section 3 , an intelligent lighting design was generated, setting the necessary parameters to the workflow shown in the previous section. Fig. 8 shows the configuration in the annual lighting calendar. The incoming data are:

- Range historic pedestrian/traffic flow: it is necessary to select a date range to choose the historic pedestrian/traffic flow to be considered for the detection of flow patterns. In this case, a provisional range of thirteen days was established during April 2013 (15/04/2013 to 28/04/2013).

- Weather: if the system user wants the lights to be turned on or off before or after the provisions, the weather must be taken into account. The system will consider the weather at the time of simulation provided by updating the on/off hours according to the weather forecast obtained on the dates indicated in the simulation.

- Maximum time weather: maximum time that the luminaires can be turned on/off before/during/after dawn and dusk. It will establish a maximum time of $30 \mathrm{~min}$.

- Limits of pedestrian/traffic flow: represent the thresholds. The minimum number of pedestrians indicates the minimum brightness level that will be maintained, and the maximum means that a maximum brightness level will be maintained. The lower limit is established at 10 pedestrians and the upper limit is set at 1700.

- Limits of luminous flux: minimum and maximum level of allowed lumens (maximum level cannot be set to a higher than permitted level of each installation). In this case the lower limit is set to $300 \mathrm{~lm}$ and the upper limit to $3000 \mathrm{~lm}$.

- Enable spending limit: if the system user wants to set a maximum estimated expenditure. For the results reported in the simulation, no approximate maximum expenditure per year is set.

The results of generating an intelligent lighting calendar by introducing these previous parameters are shown in Fig. 9. The upper panels show the data of pedestrian flow over two consecutive weeks (one week in purple and the other in blue). After applying ANOVA, traffic patterns are detected and rated per day, resulting in two groups: green for weekdays, and blue for weekends. The lower graphs show the generated light designs for each group of days. We can see that the weekend 


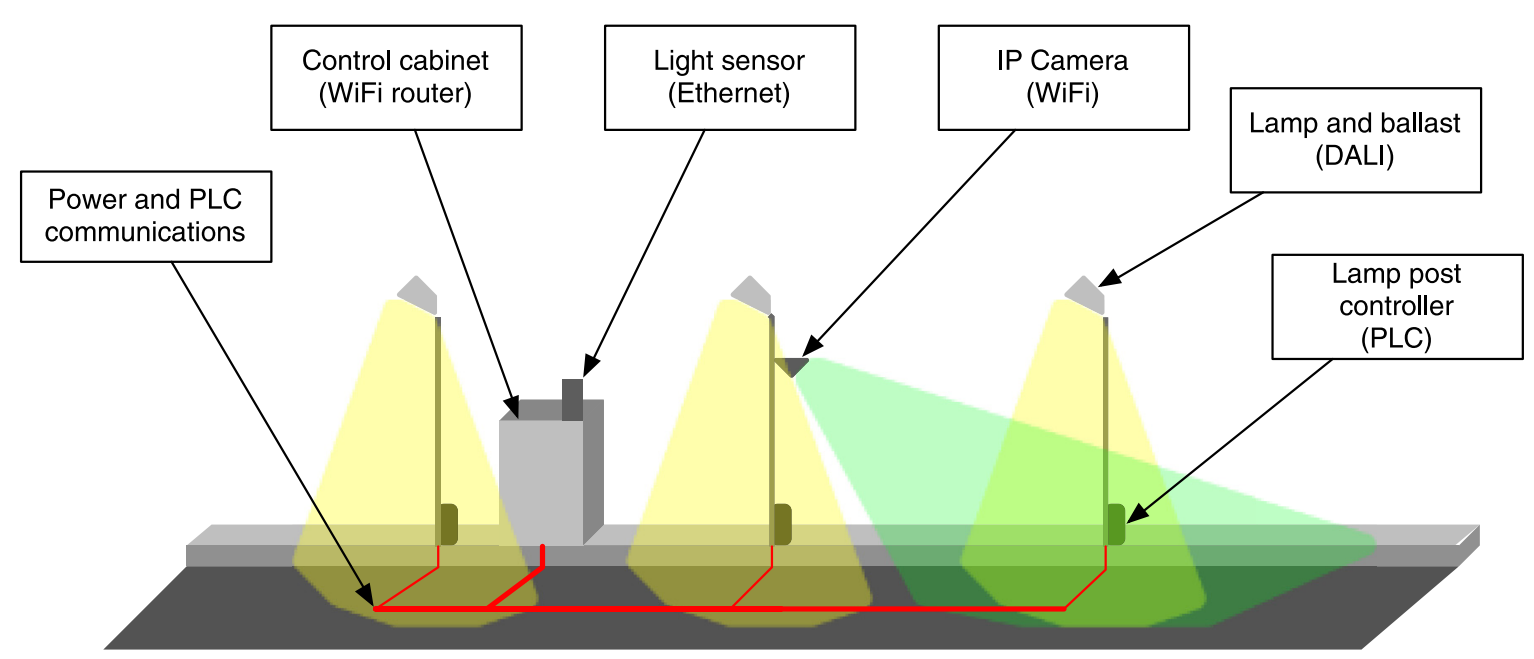

Fig. 6. Deployment in the real environment.

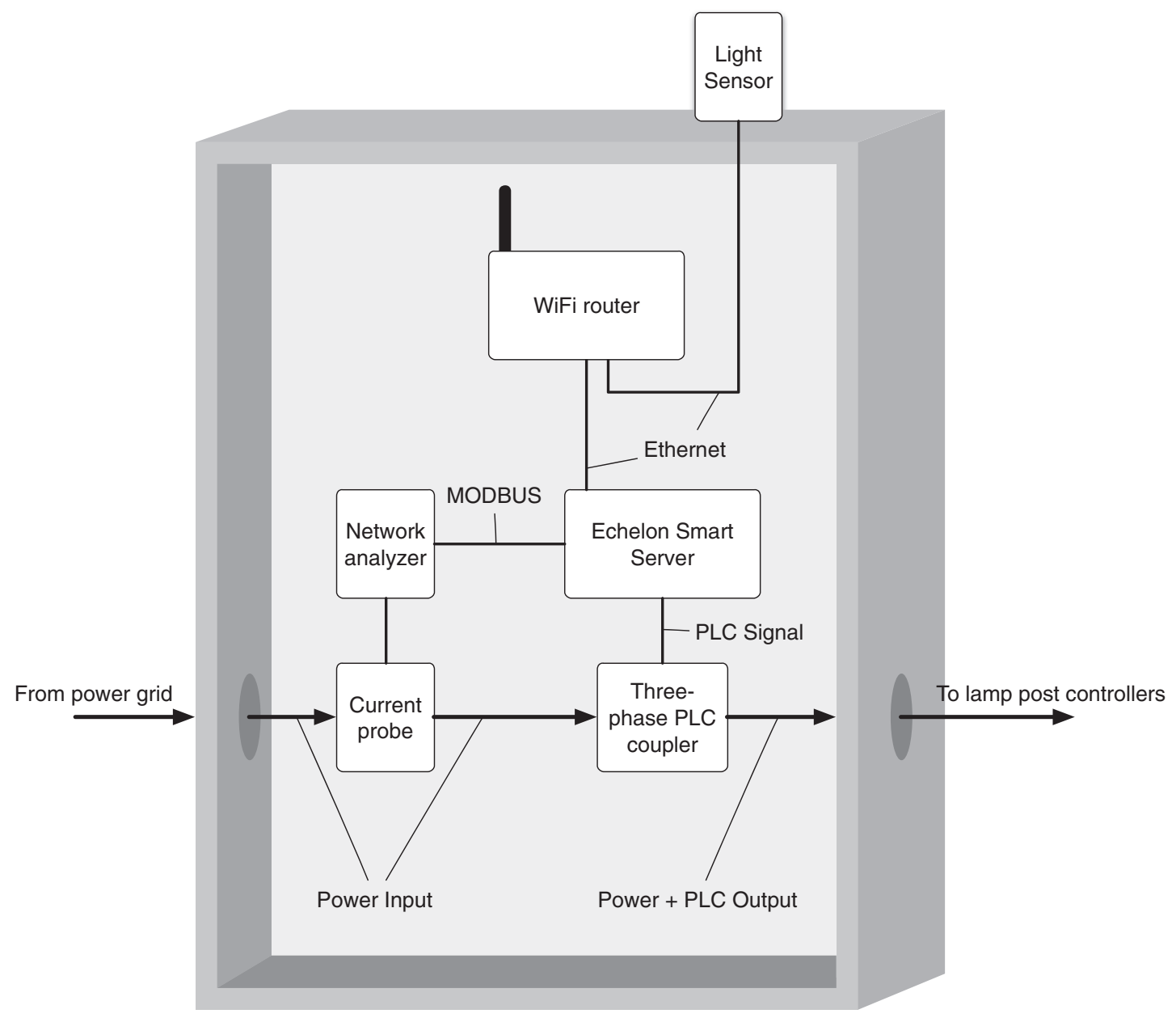

Fig. 7. Control cabinet with hardware deployed. 


\section{New Intelligent Lighting Calendar Maletin}

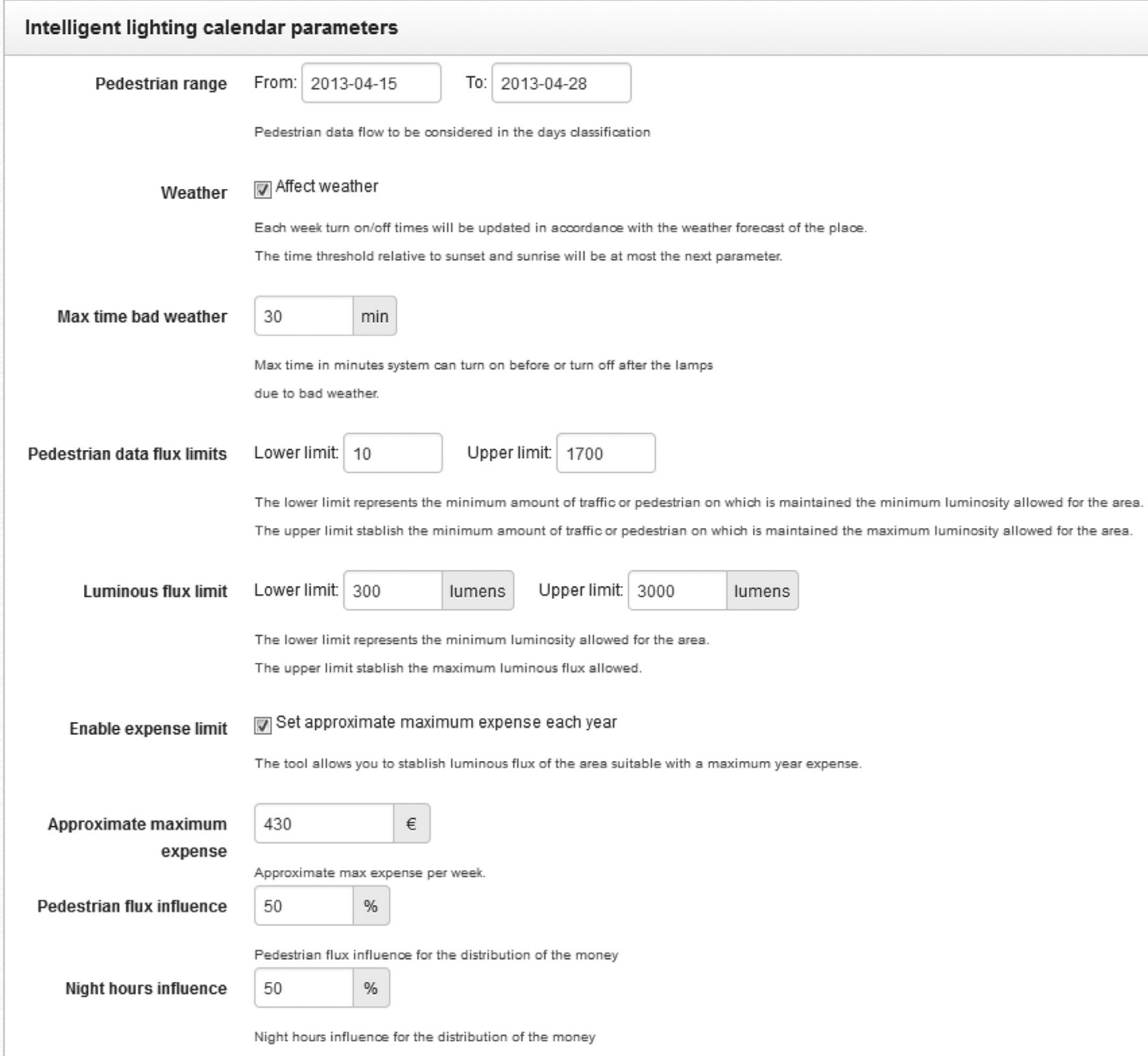

Fig. 8. Estimating annual lighting calendar.

has the same pattern while the working days have another calendar. Using the EM algorithm, hours with similar traffic are detected, adjusting a luminosity level in each group.

According to the generated lighting calendar, the system shows the information for the exposed forecast. Fig. 10 shows the cost and savings for each month. The system compares the consumption with the calendar and the maximum luminosity; in this case the percentage of savings is $19.22 \%$.

We compare the final cost with the predicted cost, and the cost with maximum luminosity. In Fig. 11 we can see that the system predicts the cost with high accuracy, with variance due to the weather. Moreover, we can see that the lowest consumption is in summer, as the system automatically adjusts the luminosity according to sunrise and sunset.

The users can also configure the luminosity manually by using the system. The user can define the luminosity pattern and then assign patterns to each day. This is important so that there are some days with specific conditions that should be managed manually. Fig. 12 shows the manual configuration form.

Fig. 13 shows a new prediction of both daily consumption and the annual calendar designed for a different configuration. The green line represents the expense of having all lights at full brightness during nighttime hours. The blue area represents the estimated model to the application of consumption. In this case, an approximate savings of $25 \%$ is achieved while maintaining the maximum light intensity at peak traffic and pedestrian flows. 


\section{Days classification}

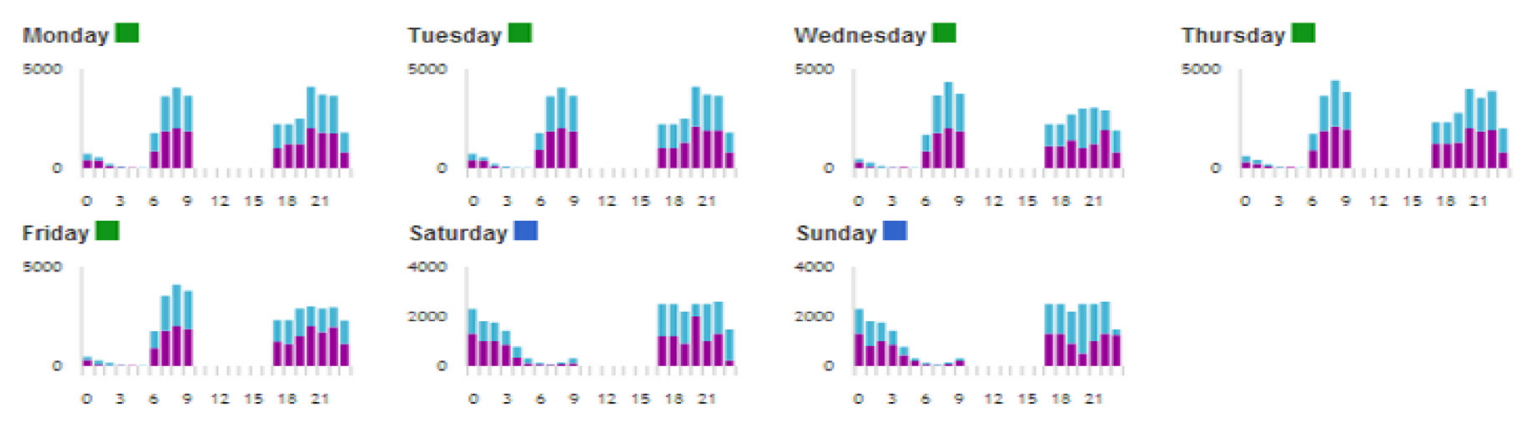

Daily lighting generated configurations

Daily luminous design "dld1int23456"

\begin{tabular}{|c|c|}
\hline Slots & Luminous flux \\
\hline $20: 00: 00-23: 00: 00$ & $2300 \mathrm{Im}$ \\
\hline $17: 00: 00-20: 00: 00$ & $1371.3 \mathrm{Im}$ \\
\hline $06: 00: 00-07: 00: 00$ & $1104.78 \mathrm{Im}$ \\
\hline $00: 00: 00-02: 00: 00$ & $515.65 \mathrm{Im}$ \\
\hline 07:00:00 - 10:00:00 & $2300 \mathrm{Im}$ \\
\hline $02: 00: 00-06: 00: 00$ & $330.21 \mathrm{Im}$ \\
\hline
\end{tabular}

Daily luminous design "dld1int71"

\begin{tabular}{|l|l|}
\hline Slots & Luminous flux \\
\hline $03: 00: 00-04: 00: 00$ & $926.08 \mathrm{Im}$ \\
\hline $00: 00: 00-01: 00: 00$ & $1343.47 \mathrm{Im}$ \\
\hline $05: 00: 00-10: 00: 00$ & $363.69 \mathrm{Im}$ \\
\hline $23: 00: 00-00: 00: 00$ & $926.08 \mathrm{Im}$ \\
\hline $17: 00: 00-23: 00: 00$ & $1343.47 \mathrm{Im}$ \\
\hline $01: 00: 00-03: 00: 00$ & $1071.73 \mathrm{Im}$ \\
\hline $04: 00: 00-05: 00: 00$ & $634.78 \mathrm{Im}$ \\
\hline
\end{tabular}

Fig. 9. Cluster and annual lighting calendar calculated.
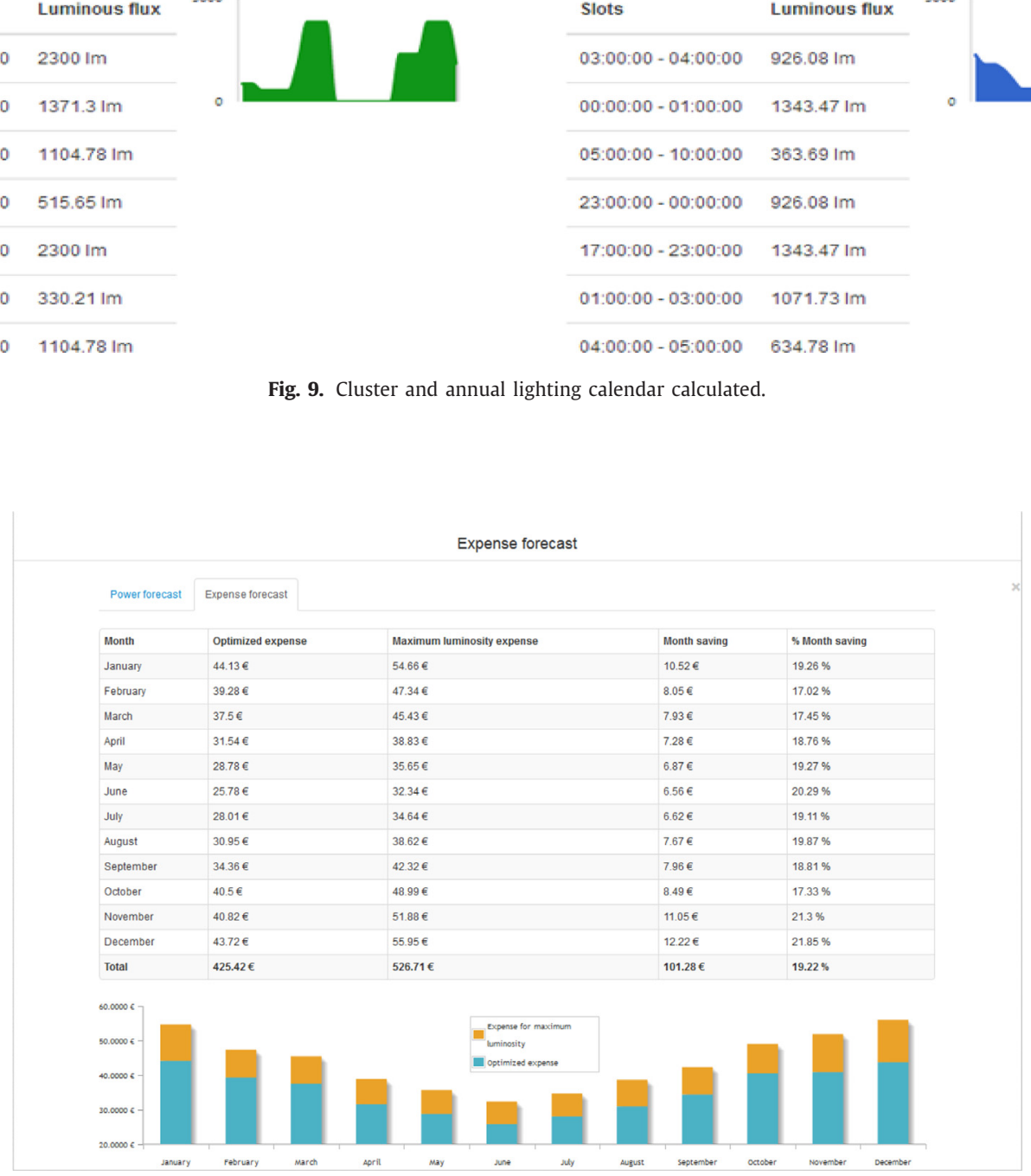

Fig. 10. Expose forecast. 


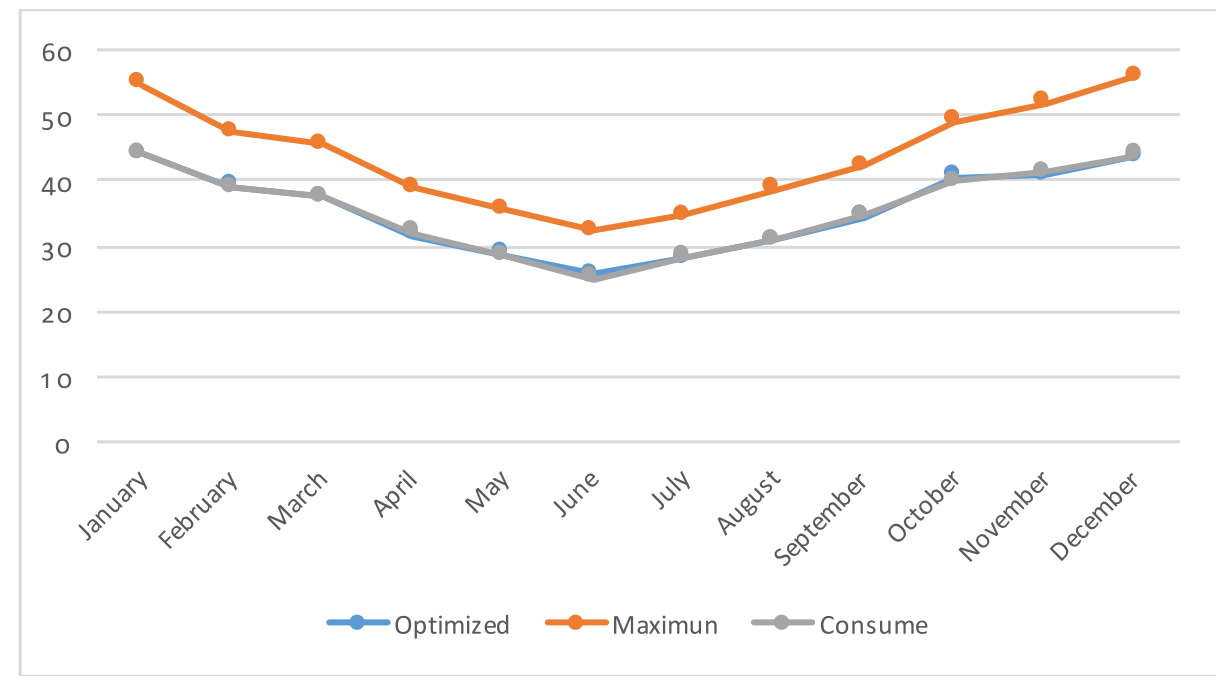

Fig. 11. Cost in euros per month for optimized, maximum and real.

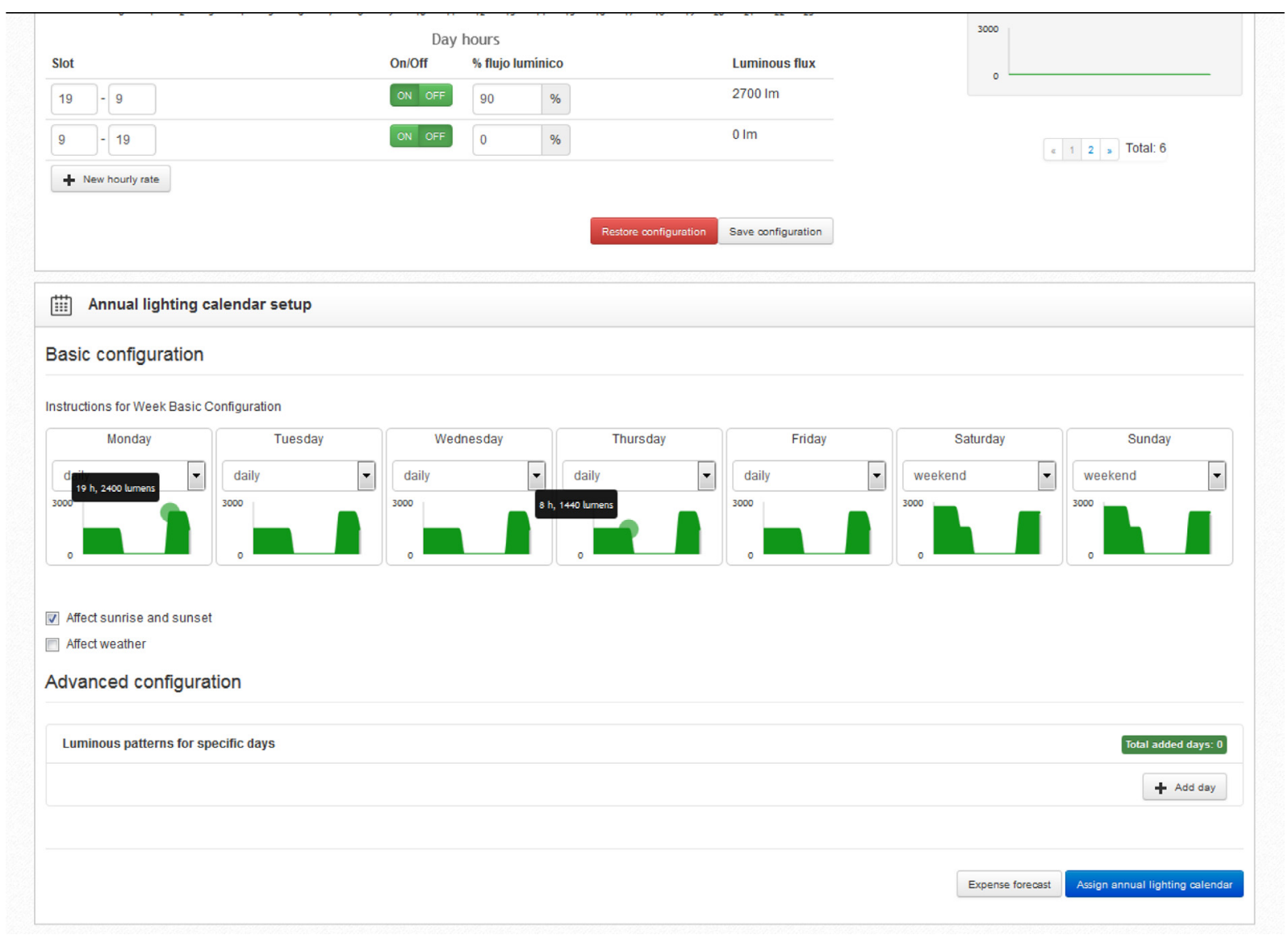

Fig. 12. Forecast. 


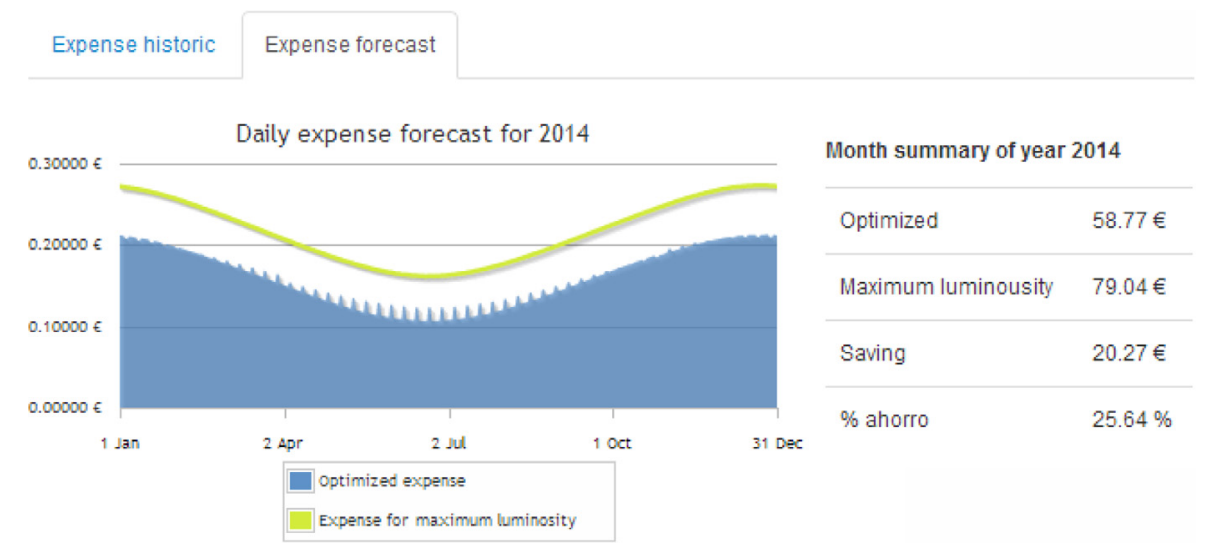

Fig. 13. Prediction of daily and annual consumption.

\section{Conclusions}

The system is able to set lighting schedules for all public controlled lighting installations. Consequently, this measure contributes to energy savings achieved by regulating light intensity. The user can define their own light patterns, which refers to the hours during which the lights are on, and at what level of brightness. Likewise, it is possible to assign different light patterns to each day.

Furthermore, not only can the lighting schedule be established according to the user preferences, but the system has the necessary mechanisms to automatically adjust light levels based on the traffic or pedestrian flow calculated for each zone. To achieve this, the system is based on historical information of people flow and classifies days to find patterns of pedestrian/traffic flow. Based on these patterns, the system establishes an appropriate lighting design for each type of day. In this sense, we have applied intelligent techniques and algorithms (ANOVA, EM clustering technique, MLP) correctly and a process that fuses everything together to determine the final lighting schedule. Furthermore, we have also presented a distribution algorithm that reduces spending and complies with the minimum luminosity and brightness levels at all times. The use of MLP networks to predict luminosity based on power, or vice versa, is performed for the sections in which the luminosity is not linear. Each model of luminaire can light to a minimum power; moreover, the expenditure of each luminaire can be influenced by the facility in which it is located (which may require more hardware, etc.). Thus, the system achieves a more accurate approximation of the luminosity that it is going to have with a certain power or, conversely, the cost of having the lights on with a certain luminous flux. Finally, the application also allows the user to query historical data related to the luminance calendars that have been established on site, and their use over time.

In conclusion, it is very difficult to find prototypes that are based on the historical data of pedestrian and traffic flow to adjust the luminosity of an area. The systems are often reactive, not predictive. The main reason for developing the system is to employ a prediction of pedestrian/traffic flow to generate savings in hardware. Placing a camera in the area for pedestrians and vehicles for a specified period of time is much cheaper than having a presence sensor in various luminaires that regulates the brightness depending on the passage of pedestrians and vehicles. Moreover, the constant change in light intensity emitted by the luminaires could result in a faster rate of deterioration. Based on new technologies and methods, this work has presented a new system developed under the concept of Smart Cities to provide intelligent lighting systems that can be controlled remotely and, consequently, offer a better quality of life to residents. The new system offers a distributed lighting systems to facilitate the implementation of a new infrastructure in a city; in other words, a modular architecture that is fully adaptable to current city's existing lighting systems to optimize energy consumption and its associated costs. Future work will focus on the following three aspects. (1) Add other sensors to the lamp and investigate how to use sensor fusion to further improve the intelligence level of the system. (2) Develop a system of alerts that is activated in real-time hardware: cast a light, overvoltage on the line, etc. (3) Develop new algorithms to make the lamp members cooperate with each other.

\section{Acknowledgements}

This work has been supported by project Sociedades Humano-Agente en entornos Cloud Computing (Soha+C). SA213U13. Project co-financed with Junta Castilla y León funds.

\section{References}

[1] S. Afshari, S. Mishra, A. Julius, F. Lizarralde, J.D Wason, J.T. Wen, Modeling and control of color tunable lighting systems, Energy Build. 68 (2014) 242-253 Part A. 
[2] K. Barra, D. Rahem, Predictive direct power control for photovoltaic grid connected system: an approach based on multilevel converters, Energy Convers. Manage. 78 (2014) 825-834.

[3] M. Beccali, M. Bonomolo, G. Ciulla, A. Galatioto, V Lo Brano, Improvement of energy efficiency and quality of street lighting in South Italy as an action of sustainable energy action plans. The case study of Comiso (RG), Energy 92 (Part 3) (2015) 394-408, doi:10.1016/j.energy.2015.05.003.

[4] D. Bourgeois, C. Reinhart, I. Macdonald, Adding advanced behavioural models in whole building energy simulation: a study on the total energy impact of manual and automated lighting control, Energy Build. 38 (7) (2006) 814-823.

[5] C. Carrillo, E. Diaz-Dorado, J. Cidrás, A. Bouza-Pregal, P. Falcón, A. Fernández, A. Álvarez-Sánchez, Lighting control system based on digital camera for energy saving in shop windows, Energy Build. 59 (2013) 143-151.

[6] H. Chourabi, T. Nam, S. Walker, J.R. Gil-Garcia, S. Mellouli, K. Nahon, T.A. Pardo, H.J. Scholl, Understanding smart cities: an integrative framework, in 45th Hawaii International Conference on System Sciences, 2012, pp. 2289-2297.

[7] Collobert, R., Bengio, S., Links between perceptrons, MLPs and SVMs. Proc. Int'l Conf. on Machine Learning (ICML). 2004

[8] R. Daigneau, Service Design Patterns: Fundamental Design Solutions for SOAP/WSDL and RESTful Web Services, Addison-Wesley Professional, 2013 Edición: 1.

[9] P. Davidsson, M. Boman, Distributed monitoring and control of office buildings by embedded agents, Inf. Sci. 171 (4) (2005) 293-307 13, doi:10.1016/ j.ins.2004.09.007.

[10] J.F. De Paz, D.I. Tapia, R.S. Alonso, C.I. Pinzón, J. Bajo, J.M. Corchado, Mitigation of the ground reflection effect in real-time locating systems based on wireless sensor networks by using artificial neural networks, Knowl. Inf. Syst. 34 (1) (2013) 193-197.

[11] A.P. Dempster, N.M. Laird, D.B. Rubin, Aximum likelihood from incomplete data via the EM algorithm, J. R. Stat. 39 (1977) 1-38.

[12] M. Domínguez, S. Alonso, A. Morán, M.A. Prada, J.J. Fuertes, Dimensionality reduction techniques to analyze heating systems in buildings, Inf. Sci. 294 (10) (2015) 553-564, doi:10.1016/j.ins.2014.06.029.

[13] E. García, S. Rodríguez, J.F. De Paz, J. Bajo, Intelligent lighting control system, Adv. Intell. Soft Comput. 290 (2015) 195297.

[14] J. Gómez-Romero, M.A. Serrano, M.A. Patricio, J. García, J.M. Molina, Context-based scene recognition from visual data in smart homes: an information fusion approach, J. Pers. Ubiquit. Comput. 16 (7) (2012) 835-857 (Special Issue on Sensor-driven Computing and Applications for Ambient Intelligence).

[15] S. Haykin, Neural Networks: A Comprehensive Foundation, second ed., Prentice Hall, 1998.

[16] IDAE, Instituto para la Diversificación y Ahorro de la Energía. www.idae.es/ [Last Access 12/01/2016]

[17] M.I. al Irsyad, R. Nepal, A survey based approach to estimating the benefits of energy efficiency improvements in street lighting systems in Indonesia, Renew. Sust. Energy Rev. 58 (2016) 1569-1577, doi:10.1016/j.rser.2015.12.294.

[18] L. Kaufman, P.J. Rousseeuw, Finding Groups in Data: An Introduction to Cluster Analysis, John Wiley and Sons Ltd, New York, 1990.

[19] R. Little, D Rubin, Statistical Analysis with Missing Data, Wiley \& Son, New York, 2002.

[20] R.G. Miller, Beyond ANOVA: Basics of Applied Statistics, CRC Press, 1997.

[21] S. Ping Lau, G.V. Merrett, A.S. Weddell, N.M. White, A traffic-aware street lighting scheme for Smart Cities using autonomous networked sensors, Comput. Elect. Eng. 45 (2015) 192-207, doi:10.1016/j.compeleceng.2015.06.011.

[22] H. Wang, Q. Jia, C. Song, R. Yuan, X. Guan, Building occupant level estimation based on heterogeneous information fusion, Inf. Sci. 272 (10) (2014) 145-157, doi:10.1016/j.ins.2014.02.080.

[23] D. Washburn, U. Sindhu, S. Balaouras, R. Dines, N. Hayes, L.E. Nelson, Helping CIOs understand "Smart City" initiatives, Growth 17 (2009).

[24] I. Wojnicki, S. Ernst, L. Kotulski, A.S dziwy, Advanced street lighting control, Expert Syst. Appl. 41 (4) (2014) $999-1005$ Part 1.

[25] C. Yang, S. Fan, Z. Wang, Li. W., Application of fuzzy control method in a tunnel lighting system, Math. Comput. Modell. 54 (3-4) (2011) 931-937.

[26] J. Zhang, G. Qiao, G. Song, H. Sun, J. Ge, Group decision making based autonomous control system for street lighting, Measurement 46 (1) (2013) $108-116$. 